\title{
A developmental nephron deficit in rats is associated with increased susceptibility to a secondary renal injury due to advanced glycation end-products
}

Received: 22 June 2005 / Accepted: 28 November 2005 / Published online: 23 February 2006

C) Springer-Verlag 2006

\begin{abstract}
Aims/hypothesis: The aim of this study was to investigate the effects of a secondary renal insult, due to chronic infusion of AGEs on renal function, and on early pathological markers in rats with a developmental nephron deficit. Methods: Female Wistar-Kyoto rats were fed a low-protein diet (LPD; $8.7 \%$ casein) or a normal-protein diet (NPD; 20\% casein) during pregnancy and lactation. Nephron number was estimated in 4-week-old female offspring. Male offspring were allowed to grow to 20 weeks of age, when AGEs derived from BSA (AGEBSA) or BSA was infused subcutaneously $\left(20 \mathrm{mg} \mathrm{kg}^{-1}\right.$ day $^{-1}$ ) for 4 weeks. At 24 weeks, blood pressure, renal function and circulating and renal AGEs were assessed. Real-time PCR was used to investigate early molecular markers of renal pathology. Results: As expected, maternal protein restriction led to reduced nephron endowment in LPD offspring. This alone did not affect blood pressure or lead to hyperfiltration in adulthood. However, when coupled with the secondary renal insult, the expression of the genes encoding transforming growth factor- $\beta_{1}$ and procollagen III was significantly upregulated in the kidneys. In addition, there was renal accumulation of AGEs in LPD offspring, and this was exacerbated by AGE infusion. Conclusions/interpretation: Our results demonstrate that the adult kidney with a reduced nephron endowment is more vulnerable to secondary renal insult from AGE-BSA. Since AGE formation is markedly elevated with hyperglycaemia, our findings suggest that
\end{abstract}

M. A. Zimanyi $(\bowtie) \cdot$ F. Poon · M. J. Black

Department of Anatomy and Cell Biology, Monash University, P.O. Box 13C, Clayton, VIC 3800, Australia e-mail: monika.zimanyi@med.monash.edu.au Tel.: +61-3-99052763

Fax: $+61-3-99052766$

K. M. Denton

Department of Physiology, Monash University,

Clayton, VIC, Australia

J. M. Forbes $\cdot$ V. Thallas-Bonke $\cdot$ M. C. Thomas

Baker Heart Research Institute,

Prahran, VIC, Australia a developmental or acquired deficit may render the kidney susceptible to diabetic renal disease.

Keywords AGE - Intrauterine growth restriction - $C c l 2$. Kidney - Low-protein diet - Maternal protein restriction . Nephron deficit · Nephron endowment $\cdot$ Secondary renal insult $\cdot \operatorname{Tg} f b 1$

Abbreviations CML: $N^{\varepsilon}$-carboxymethyllysine $\cdot$ LMW: low molecular weight - LPD: low-protein diet - NPD: normal-protein diet . WKY: Wistar-Kyoto

\section{Introduction}

Intrauterine growth restriction in humans has been shown to result in babies with low birth weight and significant nephron deficits $[1,2]$. Similarly, maternal protein restriction during pregnancy in rats results in offspring with low birth weight offspring and reduced kidney size and nephron endowment [3-5]. It has been postulated that a reduction in nephron endowment acts as a primary renal insult on the kidney, whereby compensatory mechanisms are initiated to maintain normal renal function [6]. However, with subsequent lifestyle insults (secondary insults), the kidney is thought to reach its limits of compensation, as a result of which renal function declines and onset of renal pathology occurs [6].

Accumulation of AGEs in the kidney is linked to adult renal disease [7]. AGE are a heterogeneous group of compounds, formed by the non-enzymatic reaction between reducing sugars and free amino groups of lipids, proteins or DNA. Modification of AGEs predominantly involves long-lived molecules such as extracellular matrix proteins $[8,9]$. AGEs accumulate with ageing [8-10]. However, prolonged hyperglycaemia, dyslipidaemia and oxidative stress in diabetes increases the production and accumulation of AGEs [11] in the kidney and retina and at other sites of microvascular damage [12]. AGE are thought to have an important role in the pathogenesis of the endorgan damage associated with diabetes. Their importance 
as mediators of renal injury has been amply demonstrated by studies using inhibitors of AGE formation [13-15] or dietary restriction of AGEs [16] to retard nephropathy without influencing glycaemic control. In addition, in vivo exposure of healthy adult animals to AGEs is able to generate glomerular lesions similar to those seen in diabetes, in the absence of hyperglycaemia [7, 17].

It has been proposed previously that a congenital nephron deficit is associated with an increased risk of adult renal disease [18]. However, the precise mechanisms behind this link remain to be established. In this study, we test the hypothesis that a developmental nephron deficit (primary insult) renders the kidney more susceptible to secondary renal injury induced by AGE-modified BSA administered for 4 weeks.

\section{Materials and methods}

Animal treatment

Virgin male and female normotensive Wistar-Kyoto (WKY) rats at 10 weeks of age were obtained from the Australian Resource Centre, Perth, Australia.

A low-protein diet (LPD; $8.7 \%$ casein) or a normalprotein diet (NPD; 20\% casein) was fed to the dams (eight rats per group) for 2 weeks prior to mating, during pregnancy and for a further 2 weeks after birth of the offspring. At birth, all litters were culled to eight pups and offspring were weaned at 4 weeks of age. Female offspring were perfusion-fixed at 4 weeks of age for estimation of nephron number. Male littermates were housed two or three per cage until 24 weeks of age. Between 20 and 24 weeks of age, the LPD and NPD offspring were subcutaneously infused with either non-glycated BSA or AGE-modified BSA (AGE-BSA) via an osmotic minipump (model 2ML4; Alzet, CA, USA). The preparation and composition of AGE-BSA has been described previously elsewhere [19]. This model is associated with increased circulating AGE levels comparable with those observed in advanced diabetes [20].

Animal care and experimental procedures conformed to the National Health and Medical Research Council (NH\&MRC) Code of Practice for the Care and Use of Animals for Scientific Purposes and were approved by the Animal Ethics Committee of the Departments of Biochemistry, Anatomy and Cell Biology and Microbiology of Monash University.

Perfusion fixation of 4-week-old offspring and nephron number estimation

LPD and NPD female offspring, at 4 weeks of age, were anaesthetised using sodium pentobarbitone (Nembutal, $60 \mathrm{mg} / \mathrm{ml}$; Rhone Merieux Australia, Pinkenba, QLD, Australia) at $2 \mathrm{ml} / \mathrm{kg}$ body weight and perfusion-fixed with
4\% paraformaldehyde [5]. The number of nephrons within the fixed kidneys was estimated using an unbiased physical disector/fractionator technique as previously described $[5$, 21].

Measurement of heart rate, mean arterial pressure and assessment of renal function

At 24 weeks of age, conscious mean arterial pressure and heart rate was measured for $30 \mathrm{~min}$ via a catheter in the tail artery [22]. Rats were then anaesthetised using inactin (150 mg/kg body weight), and during all subsequent procedures mean arterial pressure and heart rate were continually monitored. $\left[{ }^{3} \mathrm{H}\right]$ Inulin and $\left[{ }^{14} \mathrm{C}\right]$ para-aminohippurate were infused intravenously for measurement of GFR and effective renal blood flow [22]. Urine flow rate, renal vascular resistance and filtration fraction were also calculated [22]. On completion of the renal function studies, whilst the rats were still anaesthetised, the left kidneys were excised and snap-frozen in liquid nitrogen for later analysis of total collagen content or mRNA expression using real-time PCR. The right kidneys were perfusion-fixed using 4\% paraformaldehyde (ProSciTech, Thuringowa, QLD, Australia) in $0.1 \mathrm{~mol} / 1$ phosphate buffer at a pressure of $150 \mathrm{mmHg}$.

Quantitation of circulating AGE fluorescence and glycated haemoglobin

A $1 \mathrm{ml}$ sample of blood was drawn from the tail vein of conscious, restrained rats at 20 weeks of age (prior to subcutaneous infusion) and again at 24 weeks of age. The blood was centrifuged at $4{ }^{\circ} \mathrm{C}$ and the red blood cells and plasma were collected.

Low molecular weight (LMW) AGE fluorescence was assayed as described previously [15]. LMW fluorescence was expressed as arbitrary units, defined by the ratio of the area under the fluorescence curve divided by the area under the $A_{280}$ curve, normalised to the values derived following exhaustive enzymatic hydrolysis of AGE albumin [9].

Glycated haemoglobin levels before and after AGE infusion were quantitated by HPLC [23].

\section{Measurement of total renal collagen and collagen-associated AGE fluorescence}

Total collagen content was determined by measuring the hydroxyproline concentration after $24 \mathrm{~h}$ of pepsin digestion and acid hydrolysis $\left(6 \mathrm{~mol} / \mathrm{l} \mathrm{HCl}\right.$ for $24 \mathrm{~h}$ at $\left.110^{\circ} \mathrm{C}\right)$ using the procedure of Stegemann and Stalder [24].

Collagen-associated AGE fluorescence was determined in 24-h acid-hydrolysed collagen preparations following sequential extractions with $1.0 \mathrm{~mol} / 1 \mathrm{NaCl}, 0.5 \mathrm{~mol} / \mathrm{l}$ acetic acid and delipidation with chloroform:methanol (1:2) by 
flow injection, as described above [25]. To correct for differences in the collagen content of samples, fluorescence intensity was expressed as the percentage of fluorescence per mg renal collagen.

\section{Real-time RT-PCR}

RNA was isolated from kidneys using Trizol reagent (Life Technologies, Gaithersburg MD, USA). cDNA was synthesised with a reverse transcriptase reaction (Superscript First Strand Synthesis System for RT-PCR; Life Technologies) using the extracted total RNA. An aliquot $(6 \mu \mathrm{g})$ of the resulting cDNA was used in the real-time PCR amplifications. To quantitate the expression of the genes encoding transforming growth factor $\beta_{1}(T g f b l)$, transforming growth factor, beta induced (Tgfbi; previously known as $\beta i g-H 3)$, chemokine ( $\mathrm{C}-\mathrm{C}$ motif) ligand 2 ( $\mathrm{Ccl}$; previously known as $M C P-1$ ), fibronectin, procollagen III, procollagen IV, mRNA levels were measured by real-time quantitative PCR using the TaqMan system (Applied Biosystems, Foster City, CA, USA). To assess genomic DNA contamination, controls without reverse transcriptase were included. Primers and probes (Table 1) were constructed using Primer Express (ABI Prism 7700; Perkin-Elmer, Foster City, CA, USA).

Each sample was run and analysed in triplicate. The samples from the control kidneys were used as the calibrator and given an arbitrary value of 1 , and the treatment groups were compared with this calibrator [26].
Immunohistochemical localization of renal AGE accumulation

Localization of renal AGEs was determined by immunohistochemistry using a polyclonal AGE antibody as previously described [27], whose main epitope for recognition is the non-fluorescent AGE $N^{\varepsilon}$-carboxymethyllysine (CML). Briefly, perfusion-fixed right kidneys were embedded in paraffin wax, sectioned at $4 \mu \mathrm{m}$ thickness, and de-waxed prior to quenching of endogenous peroxidases using 3\% $\mathrm{H}_{2} \mathrm{O}_{2} / \mathrm{PBS}$. Non-specific proteins were blocked using protein blocking agent (Thermo Shandon, Pittsburgh, PA, USA). The primary antibody, diluted in $2 \%$ BSA in PBS (1:500), was applied and allowed to bind overnight. The sections were subsequently incubated with biotinylated goat ant-rabbit IgGs (Vector Laboratories, Burlingame, CA, USA; 1:500 dilution), avidin-biotin complex reagent (Vector Laboratories) were applied and the binding sites were identified using diaminobenzidine (Sigma Chemical, St Louis, MO, USA) diluted in hydrogen peroxide and counterstained with haematoxylin. The primary antibody was omitted from the negative controls.

Statistical analysis

All data are expressed are mean \pm SD unless otherwise stated. Before statistical analysis, all data were tested for normality (Gaussian distribution). All data were found to be normally distributed. Changes in body weight over time were analysed using two-way ANOVA with repeated measures using GraphPad Prism (version 4.00; GraphPad Software, San Diego, CA, USA). Two-way ANOVA was

Table 1 Primer and probe sequences used in real-time RT-PCR

\begin{tabular}{|c|c|}
\hline Gene & Sequence \\
\hline \multicolumn{2}{|c|}{ Forward primer sequence } \\
\hline $\operatorname{Tg} f b 1$ & 5'-TTGCCCTCTACAACCAACACAA \\
\hline$T g f b i$ & 5'-TCTGCATTGAAAACAGCTGCAT \\
\hline $\mathrm{Ccl} 2$ & 5'-CTCAGCCAGATGCAGTTAATGC \\
\hline Fibronectin & 5'-CATGGCTTTAGGCGAACCA \\
\hline Procollagen III & 5'-GGAAAAGATGGATCAAGTGGACAT \\
\hline Procollagen IV & 5'-GGCGGTACACAGTCAGACCAT \\
\hline \multicolumn{2}{|c|}{ Reverse primer sequence } \\
\hline $\operatorname{Tg} f b 1$ & 5'-GGCTTGCGACCCACGTAGTA \\
\hline$T g f b i$ & 5'-CAGCATCCGGTCCATGGT \\
\hline $\mathrm{Ccl} 2$ & 5'-AGCCGACTCATTGGGATCAT \\
\hline Fibronectin & 5'-CATCTACATTCGGCAGGTATGG \\
\hline Procollagen III & 5'-GAGCCCTCAGATCCTCTTTCAC \\
\hline Procollagen IV & 5'-GGAATAGCCGATCCACAGTGA \\
\hline \multicolumn{2}{|c|}{ TaqMan probe sequence } \\
\hline $\operatorname{Tg} f b 1$ & FAM5'-CCGGGTGCTTCCGCATCACC-TAMRA \\
\hline$T g f b i$ & FAM5'-CAGGGTCCCATACCGTCCCTTCTTGT-TAMRA \\
\hline $\mathrm{Ccl} 2$ & FAM5'-CACCTGCTGCTACTCATTCACTGGCAA-TAMRA \\
\hline Fibronectin & FAM5'-CCCCGTCAGGCT-MGBNFQ \\
\hline Procollagen III & FAM5'-CCCATTGGACCACCAGGGCCTC-TAMRA \\
\hline Procollagen IV & FAM5'-CAGTGCCCTAACGGT-MGBNFQ \\
\hline
\end{tabular}




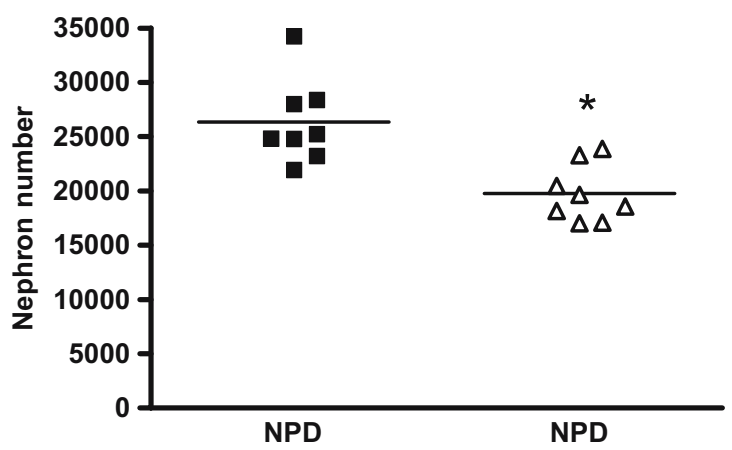

Fig. 1 Stereological estimates of nephron number at 4 weeks of age in the left kidney of female LPD and NPD offspring. Symbols represent individual animals and the lines represent the mean number of glomeruli per group. ${ }^{*} p=0.0014$ vs NPD offspring

applied to data at 24 weeks of age to determine whether there were specific effects due to maternal protein restriction and/or infusion of AGEs or whether there was an interaction effect whereby the offspring exposed to maternal protein restriction responded differently to AGE treatment compared with the NPD offspring. In data analysed with two-way ANOVA, the factors were represented as diet (D; LPD or NPD), AGE treatment (T; BSA or AGE$\mathrm{BSA})$ and interaction $(\mathrm{D} \times \mathrm{T})$.

When comparing two groups, unpaired Student's $t$-test was used. Statistical significance was accepted at the level of $p<0.05$.

\section{Results}

Weight gain, food intake and litter size of dams

Weight gain of the dams during pregnancy was not altered by the protein composition of the diets (LPD, 93.8 $\pm 3.0 \mathrm{~g}$; NPD, $97.5 \pm 5.9 \mathrm{~g}$ ) and the average daily food consumption was not significantly different between groups (LPD, $17.9 \pm 1.3 \mathrm{~g} /$ day; NPD, $19.4 \pm 0.4 \mathrm{~g} /$ day). Litter size was not affected by the protein composition in the maternal diet (LPD, 12.6 \pm 0.6 pups; NPD, 12.7 \pm 1.0 pups). Birth weights of all liveborn male and female LPD offspring $(3.84 \pm 0.05 \mathrm{~g})$ were significantly reduced $(p=0.009)$ when compared with the NPD offspring $(4.0 \pm 0.04 \mathrm{~g})$.
Nephron endowment at weaning

At 4 weeks of age, the LPD offspring exhibited a $25 \%$ reduction in nephron number when compared with NPD offspring ( $p=0.001)$ (Fig. 1).

Body weight and kidney weight in adult offspring

At 24 weeks of age, body weights and fixed kidney weights were significantly reduced in the LPD offspring $\left(p_{\mathrm{D}}<0.0001\right)$, but were not altered as a result of AGE infusion (Table 2).

Circulating LMW AGEs and glycated haemoglobin levels

There was no significant difference in circulating LMW AGE fluorescence between the LPD and NPD offspring at 20 weeks of age, before induction of the secondary renal insult (Table 3). After infusion of AGE-BSA, LMW AGE fluorescence was significantly elevated $(p<0.0001)$. However, the level of glycated haemoglobin was not significantly different between the NPD and LPD treatment groups before or after AGE-BSA infusion (Table 3).

Renal collagen-associated AGE fluorescence and renal AGE content

Renal collagen-associated AGE fluorescence was significantly increased $\left(p_{\mathrm{T}}=0.0004\right)$ following a 4 -week infusion of AGE-BSA in both LPD and NPD animals (Fig. 2). Interestingly, renal collagen-associated AGE fluorescence was also significantly greater $\left(p_{\mathrm{D}}<0.0001\right)$ in the LPD offspring receiving BSA alone, compared with NPD controls. These differences were confirmed immunohistochemically by detection of increased staining for nonfluorescent CML in animals infused with AGE-BSA (Fig. 3). Of particular note, in the LPD animals receiving AGE-BSA there was prominent immunohistochemical localisation of CML immunostaining within the glomeruli that was not observed in the other groups (Fig. 3).

Table 2 Body weight, kidney weight, conscious heart rate, conscious mean arterial pressure and total glomerular filtration rate in LPD and NPD offspring at 24 weeks of age

\begin{tabular}{lccccc}
\hline & NPD/BSA & NPD/AGE & LPD/BSA & LPD/AGE & $P$ value \\
\hline Body weight (g) & $408.9 \pm 20.2$ & $412.7 \pm 17.1$ & $387.6 \pm 23.9$ & $374.8 \pm 29.0$ & $p_{\mathrm{D}}<0.0001$ \\
Kidney weight (mg) & $1674 \pm 83$ & $1683 \pm 99$ & $1505 \pm 111$ & $1509 \pm 117$ & $p_{\mathrm{D}}<0.0001$ \\
Heart rate (beats/min) & $313 \pm 36$ & $276 \pm 18$ & $307 \pm 22$ & $316 \pm 28$ & $p_{\mathrm{D}}<0.05$ \\
Conscious MAP (mmHg) & $118 \pm 7$ & $117 \pm 8$ & $115 \pm 3$ & $115 \pm 8$ & $p_{\mathrm{D} \times \mathrm{T}}<0.005$ \\
Total GFR (ml/min) & $1.92 \pm 0.37$ & $1.65 \pm 0.29$ & $1.58 \pm 0.25$ & $1.49 \pm 0.45$ & $p_{\mathrm{D}}<0.05$ \\
\hline
\end{tabular}

$n=10$ or more animals per group

Data were analysed by two-way ANOVA with factors diet (D: LPD or NPD), AGE treatment (T: BSA or AGE-BSA) and diet $\times$ AGE treatment interaction $(\mathrm{D} \times \mathrm{T})$. GFR, Glomerular filtration rate $M A P$, Conscious mean arterial pressure 
Table 3 Plasma LMW AGE-fluorescence and glycated haemoglobin levels before (20 weeks of age) and after AGE or non-glycated BSA infusion (24 weeks of age) in LPD and NPD offspring

\begin{tabular}{lllll}
\hline & NPD/BSA $(n=14)$ & NPD/AGE $(n=13)$ & LPD/BSA $(n=13)$ & LPD/AGE $(n=14)$ \\
\hline LMW fluorescence $(\%)$ & & & & \\
Before treatment & $6.24 \pm 0.89$ & $6.37 \pm 0.85$ & $5.87 \pm 0.60$ & $6.12 \pm 0.68$ \\
After treatment & $6.15 \pm 0.72$ & $7.34 \pm 0.69^{\mathrm{a}}$ & $5.95 \pm 0.29$ & $6.96 \pm 0.43^{\mathrm{a}}$ \\
Glycated haemoglobin (\%) & & & & $2.16 \pm 0.28$ \\
Before treatment & $2.40 \pm 0.57$ & $2.56 \pm 0.70$ & $2.37 \pm 0.53$ & $2.18 \pm 0.11$ \\
After treatment & $2.16 \pm 0.37$ & $2.29 \pm 0.29$ & $2.14 \pm 0.16$ & \\
\hline
\end{tabular}

${ }^{\mathrm{a}} p<0.0001$ vs baseline

Mean arterial pressure and heart rate

At 24 weeks of age, conscious mean arterial pressures were not significantly affected by maternal protein diet or AGE infusion (Table 2). In addition, it is to be noted that there were no significant differences between conscious (Table 2) and anaesthetised mean arterial pressures (Fig. 4a) within the experimental groups. Heart rates were increased in response to AGE-BSA infusion in LPD offspring but reduced in the NPD offspring, compared with animals receiving BSA alone.

\section{Renal function}

Renal vascular resistance was significantly elevated in rats infused with AGE-BSA compared with BSA controls $\left(p_{\mathrm{T}}=0.04\right)$, with the highest renal vascular resistance recorded in the LPD/AGE group (Fig. 4c). There was no effect of maternal protein restriction or AGE-BSA infusion on effective renal blood flow adjusted for kidney weight (Fig. 4b).

Total glomerular filtration rate was significantly reduced $\left(p_{\mathrm{D}}=0.023\right)$ in LPD offspring when compared with NPD offspring (Table 2), but when adjusted for kidney weight there was no effect (Fig. 4d). Filtration fraction was significantly elevated in the LPD offspring when compared with the NPD offspring ( $p_{\mathrm{D}}=0.03$; Fig. $\left.4 \mathrm{e}\right)$. There was no

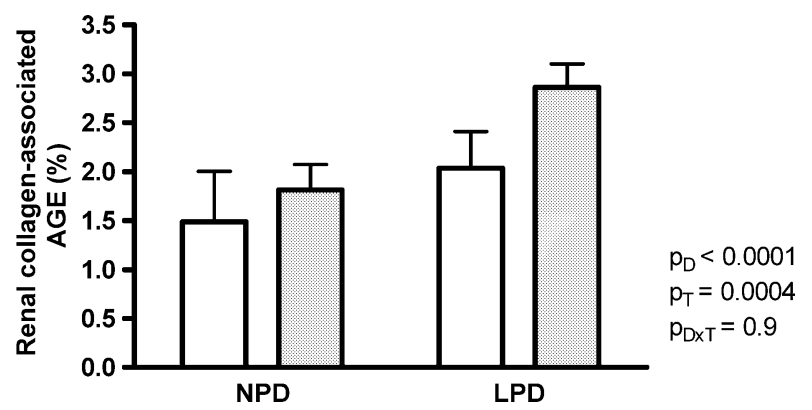

Fig. 2 Renal collagen-associated AGE fluorescence at 24 weeks of age in LPD and NPD offspring infused with either non-glycated BSA (open bars) or AGEs (shaded bars) from 20-24 weeks of age. Data are percentage fluorescence per mg collagen; $n=6$ or more per group. Data were analysed by two-way ANOVA with diet (D: LPD or NPD), AGE treatment (T: BSA or AGE-BSA) and $\operatorname{diet} \times$ AGE treatment interaction $(\mathrm{D} \times \mathrm{T})$ as factors effect of AGE-BSA infusion on filtration fraction. Urine flow rate was significantly reduced in LPD offspring when compared with NPD offspring $\left(p_{\mathrm{D}}=0.03\right.$; Fig. $\left.4 \mathrm{f}\right)$; the slowest average urine flow rate was recorded in the LPD/ AGE group.

Expression of early molecular markers of renal injury

\section{$\operatorname{Tg} f b 1$}

The mRNA levels of $T g f b 1$ were significantly elevated in the kidneys of LPD offspring when compared with NPD offspring $\left(p_{\mathrm{D}}=0.002\right.$; Fig. 5a). Furthermore, there were elevations in renal $T g f b 1 \mathrm{mRNA}$ levels in LPD offspring infused with AGE-BSA that did not occur in the NPD offspring infused with AGE-BSA ( $p_{\mathrm{D} \times \mathrm{T}}=0.02$; Fig. $5 \mathrm{a}$ ).

\section{$T g f b i$}

Real-time PCR demonstrated a significant elevation $\left(p_{\mathrm{D}}=0.005\right)$ in the levels of $T g f b i$ mRNA in all LPD offspring, with no specific effects observed following an AGE-BSA infusion or interaction between maternal diet and AGE-BSA infusion (Fig. 5b).

\section{Ccl2}

There were no significant effects on the level of renal $C c l 2$ mRNA expression as a result of maternal protein restriction or AGE-BSA infusion (Fig. 5c). When compared with the NPD offspring, there was an increase in the $C c l 2$ mRNA level in the kidneys of the LPD offspring in response to AGE infusion. However, this did not quite reach statistical significance $\left(p_{\mathrm{D} \times \mathrm{T}}=0.06\right.$; Fig. $\left.5 \mathrm{c}\right)$. It is to be noted that in two of the LPD animals receiving AGE-BSA there was more than a four-fold increase in renal $C c l 2$ mRNA levels.

\section{Fibronectin}

Likewise, there was no effect of maternal diet or AGE-BSA infusion on fibronectin mRNA levels in the kidney (Fig. 5d). However, there was a trend for fibronectin 
Fig. 3 Representative photomicrographs of AGE immunostaining in the renal cortex from (a) NPD/BSA, (b) NPD/AGE, (c) LPD/BSA, (d) LPD/AGE groups and (e) negative control. Positive staining is shown as brown. Sections are counterstained with haematoxylin. Magnification $\times 200$

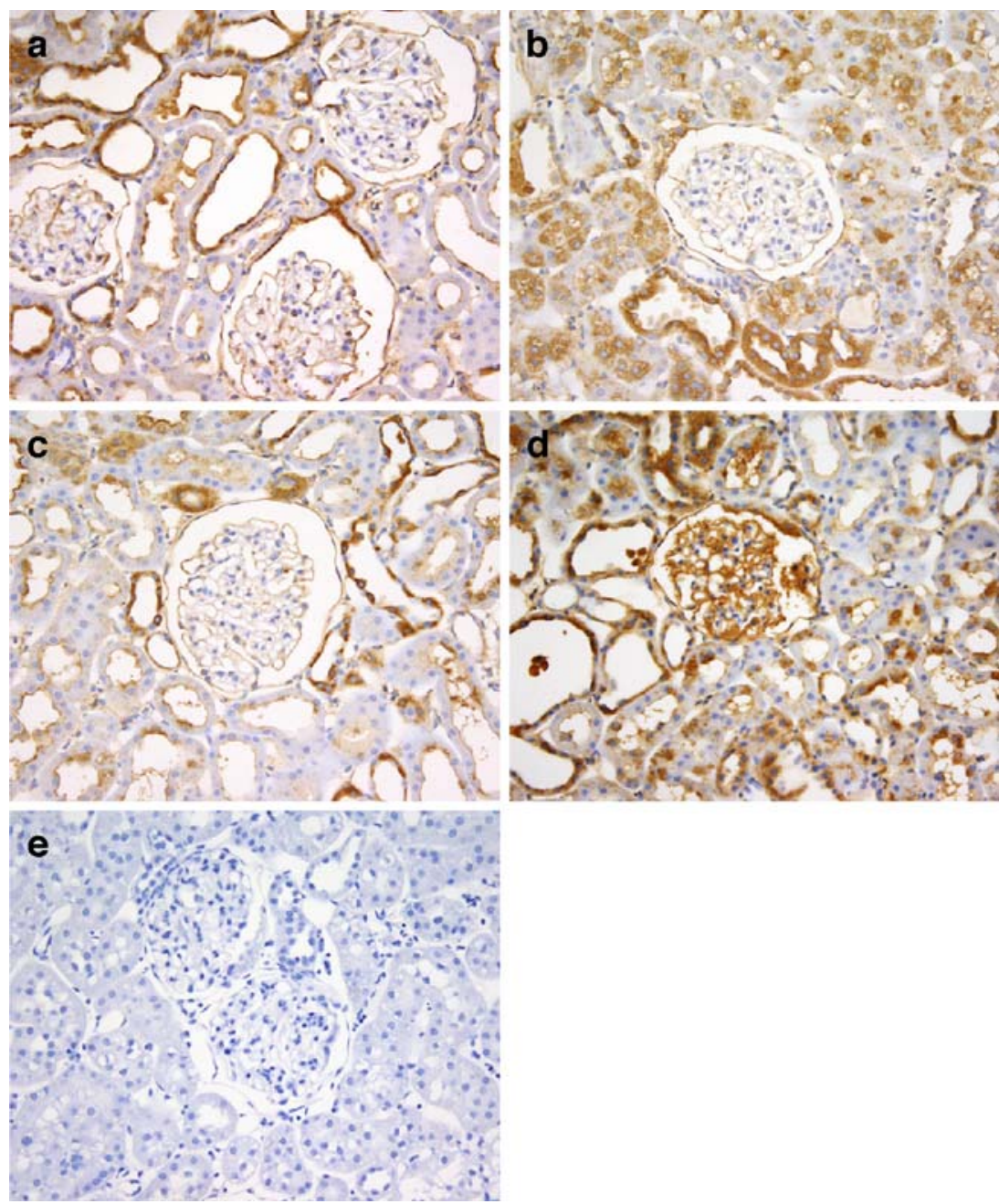

mRNA levels to be upregulated in the kidneys of the LPD offspring in response to AGE infusion, which was not observed in the NPD offspring infused with AGEs (Fig. 5d). However, this interaction between maternal diet and AGE infusion did not quite reach statistical significance $\left(p_{\mathrm{D} \times \mathrm{T}}=0.09\right)$.

\section{Procollagen III}

There was increased expression of procollagen III mRNA in LPD offspring infused with AGEs compared with those receiving BSA alone. This effect was not observed in NPD offspring infused with AGEs, as demonstrated by a significant interaction $\left(p_{\mathrm{D} \times \mathrm{T}}=0.03\right)$ between maternal diet and AGE treatment (Fig. 5e).

\section{Procollagen $I V$}

Procollagen IV mRNA levels were not significantly affected by maternal diet or AGE-BSA infusion (Fig. 5f). In addition, there was also no difference in procollagen IV
mRNA between the LPD and NPD groups in response to AGE-BSA (Fig. 5f).

\section{Total kidney collagen}

Total kidney collagen, as determined by hydroxyproline content, was not affected by maternal diet or AGE-BSA infusion (Fig. 6).

\section{Discussion}

In this study, maternal protein restriction during pregnancy and lactation led to reduced nephron endowment in the offspring. This primary insult to the kidney did not affect conscious blood pressure or lead to overt renal dysfunction. However, a secondary renal insult following a 4-week infusion of AGE-BSA resulted in differential changes in renal parameters in animals with reduced nephron numbers compared with those with a normal endowment. In particular, there was significant upregulation of gene expression of the profibrotic cytokine Tgfbl and procollagen III in the kidneys of LPD animals following an AGE 
a

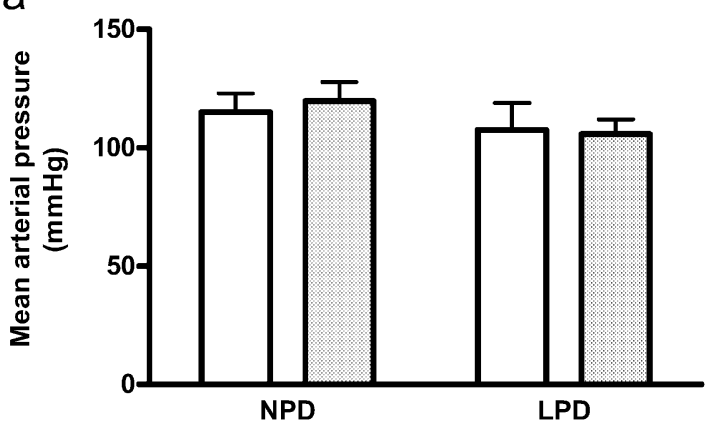

$\mathrm{p}_{\mathrm{D}}<0.0001$

$\mathrm{p}_{\mathrm{T}}=0.56$

$\mathrm{p}_{\mathrm{DxT}}=0.19$

C

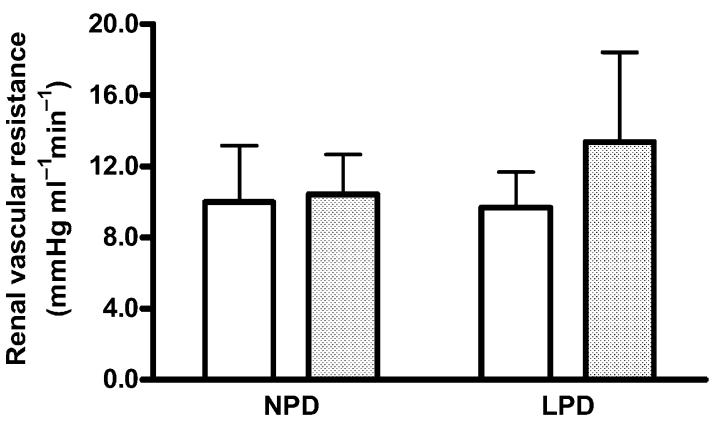

$\mathrm{p}_{\mathrm{D}}=0.17$

$\mathrm{p}_{\mathrm{T}}=0.04$

$\mathrm{P}_{\mathrm{DxT}}=0.1$

e

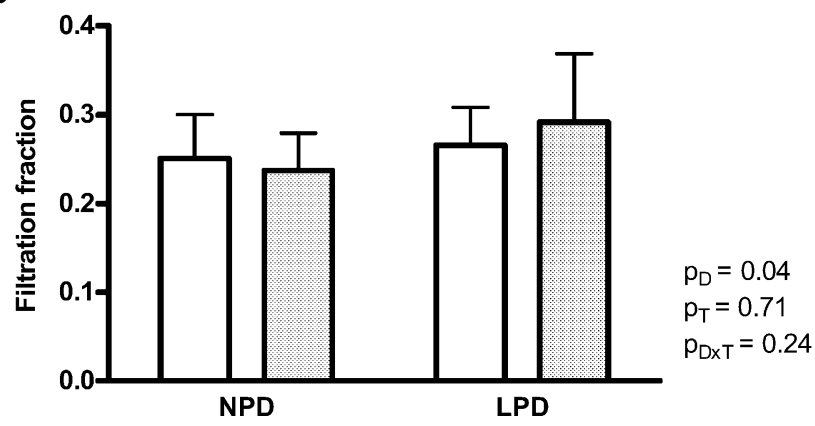

Fig. 4 Anaesthetised mean arterial pressure (a), effective renal blood flow (per g kidney weight) (b), renal vascular resistance (c), glomerular filtration rate (per g kidney weight) (d), filtration fraction (e) and urine flow rate (f) at 24 weeks of age in NPD and LPD offspring infused with non-glycated BSA (open bars) or AGEs

infusion that was not observed in NPD animals. Notably, these changes were associated with increased accumulation of fluorescent and non-fluorescent AGEs in the kidneys of animals with reduced nephron endowment. Expression of Ccl2 and fibronectin also showed similar trends in LPD offspring infused with AGEs, correlating with changes in $T g f b 1$ expression, but these did not quite reach statistical significance, reflecting large variability in these parameters.

Although maternal protein restriction during pregnancy and lactation had no major effect on renal function, kidneys from these animals were not normal. Notably, there was increased renal expression of genes for several key molecules linked to renal fibrosis and glomerulosclerosis, including $T g f b l$ and $T g f b i$, and an increased renal filtration fraction. In addition, renal AGE accumulation was significantly greater in animals with reduced nephron endowment, which was exacerbated following an infusion b



d

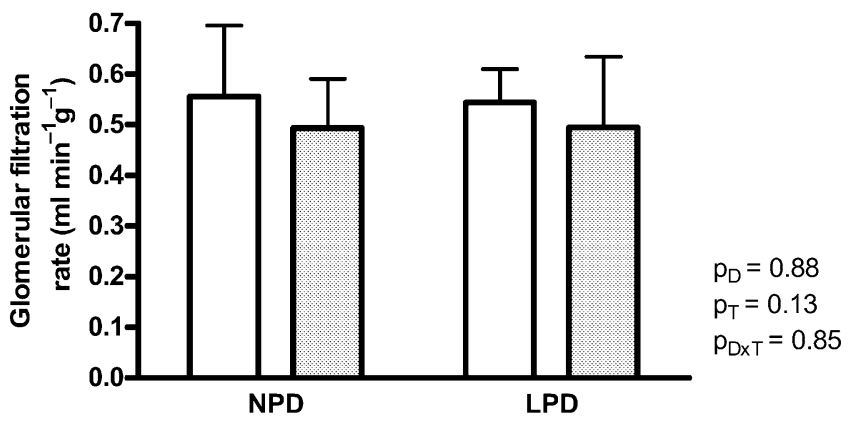

f

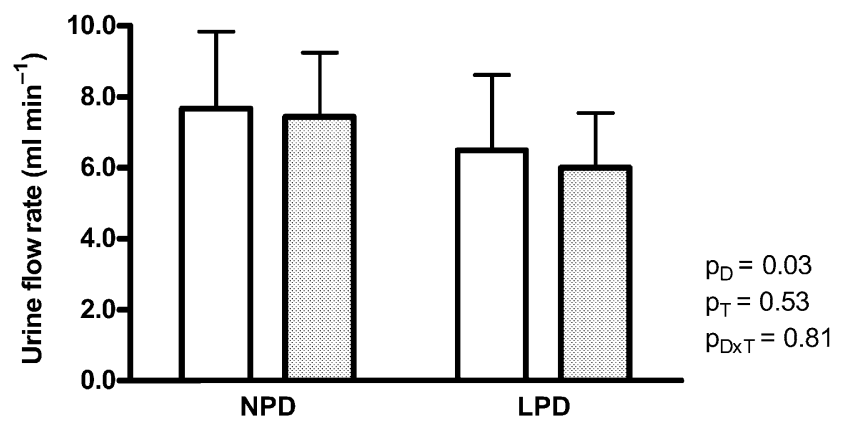

(shaded bars); $n=10$ or more animals per group. Data were analysed by two-way ANOVA with diet (D: LPD or NPD), AGE treatment (T: BSA or AGE-BSA) and diet $\times$ AGE treatment interaction $(\mathrm{D} \times \mathrm{T})$ as factors

of AGE-BSA. Of particular note was the accumulation of CML-AGEs (shown immunohistochemically) in the glomeruli of the LPD offspring infused with AGEs. To the extent that AGE accumulation is a strong predictor of adverse renal outcomes in patients with diabetes, it is possible to speculate that reduced nephron endowment may be associated with accelerated AGE accumulation, and therefore more aggressive renal disease, in the setting of diabetes. This is consistent with the Brenner hypothesis, which links the observed association of reduced nephron number with the subsequent increased risk of renal disease in adults [18]. In support of this concept, Jones et al. report exacerbated renal and glomerular hypertrophy in adult LPD rat offspring following induction of streptozotocininduced diabetes and that these glomerular abnormalities persist even when insulin is administered [28]. The factors leading to increased AGE accumulation in animals with a 
a

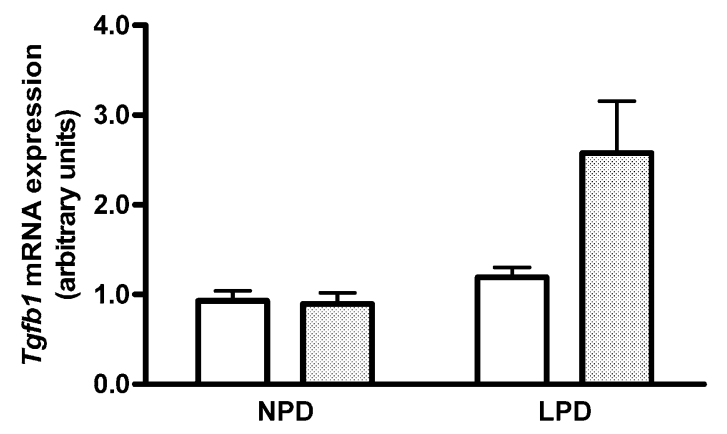

C

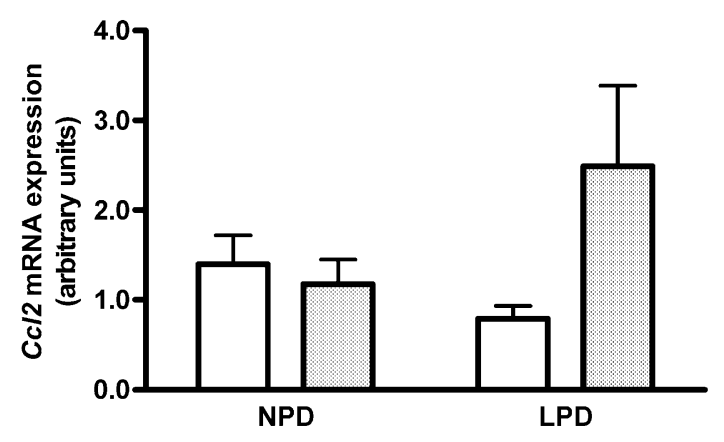

e

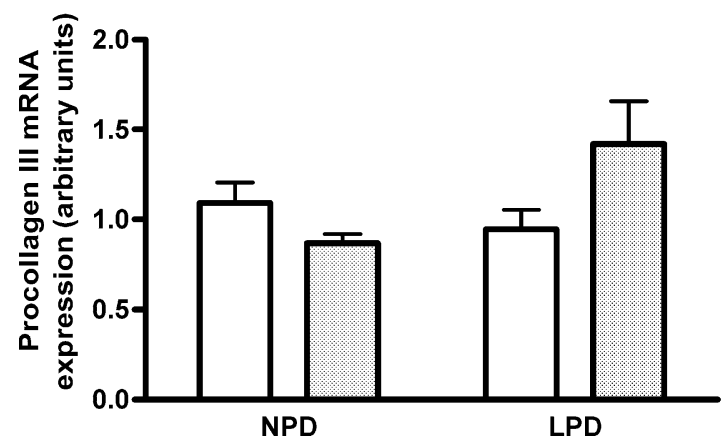

Fig. 5 mRNA expression of the genes for (a) transforming growth factor $\beta_{1}$, (b) transforming growth factor, beta induced, (c) chemokine (C-C motif) ligand 2, (d) fibronectin, (e) procollagen III and (f) procollagen IV at 24 weeks of age in NPD and LPD offspring infused with non-glycated BSA (open bars) or AGEs

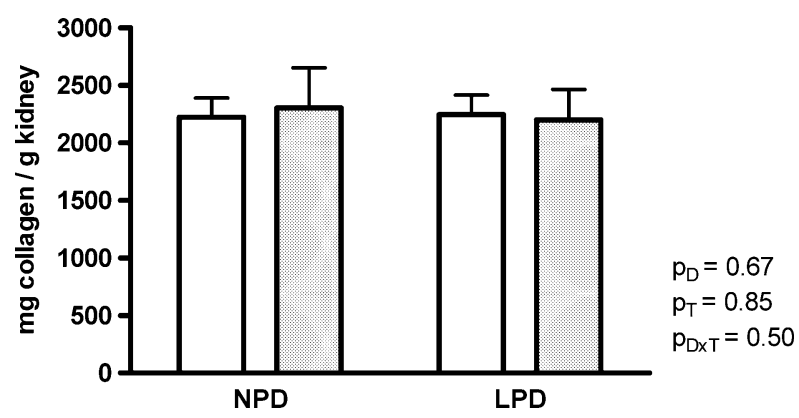

Fig. 6 Total kidney collagen at 24 weeks of age in LPD and NPD offspring infused with either non-glycated BSA (open bars) or AGEs (shaded bars); $n=7$ or 8 animals per group. Data were analysed by two-way ANOVA with diet (D: LPD or NPD), AGE treatment (T: BSA or AGE-BSA) and $\operatorname{diet} \times$ AGE treatment interaction $(\mathrm{D} \times \mathrm{T})$ as factors b

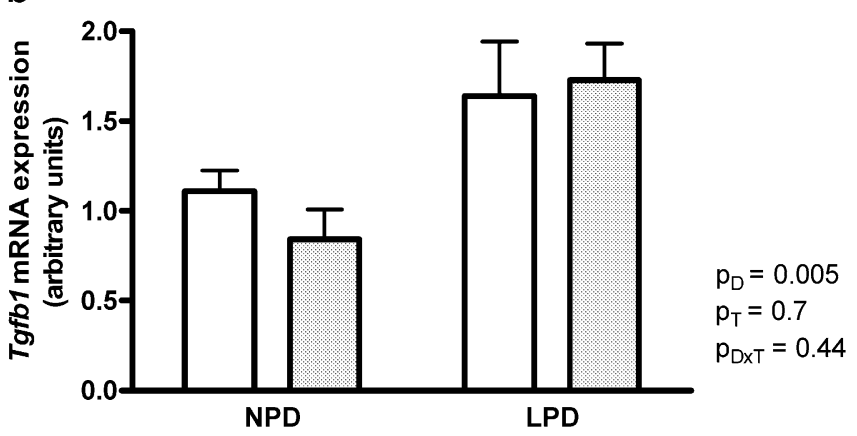

d

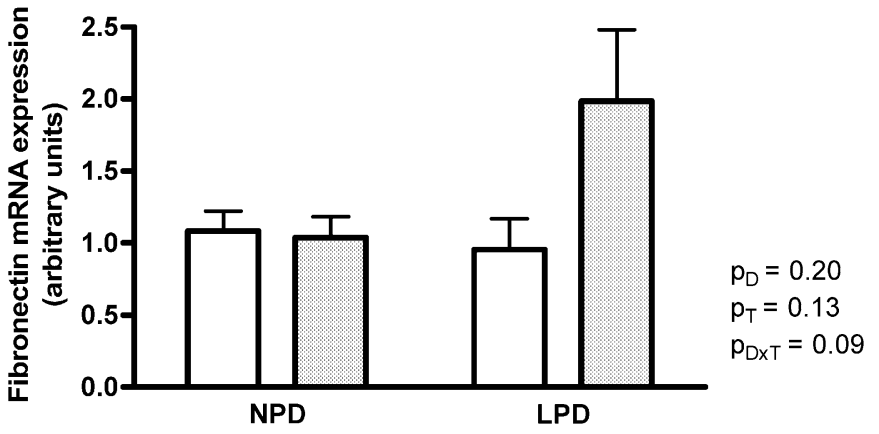

f

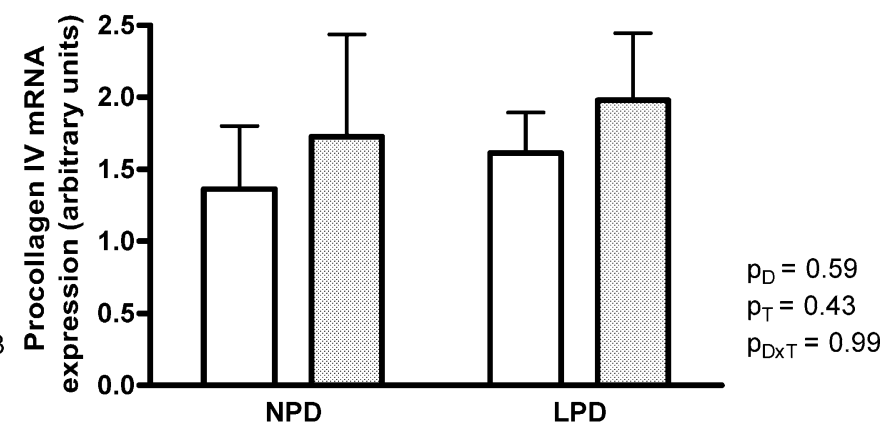

(shaded bars); $n=4$ or more animals per group. All groups are normalised to the NPD/BSA (control) group. Data were analysed by two-way ANOVA with diet (D: LPD or NPD), AGE treatment (T: BSA or AGE-BSA) and diet $\times$ AGE treatment interaction $(\mathrm{D} \times \mathrm{T})$ as factors

reduced number of nephrons remain to be established. In previous studies in diabetes, reduced renal filtration of circulating AGEs and increased AGE formation associated with hyperglycaemia have been linked to renal AGE accumulation in patients with diabetic nephropathy $[29,30]$. However, mechanisms other than GFR and dysglycaemia appear to contribute to AGE accumulation in the LPD model.

In our laboratory we have consistently shown reduced nephron endowment at the completion of nephrogenesis in offspring exposed to maternal protein restriction [5]. In this study we were unable to count the number of nephrons in the male LPD offspring at 4 weeks of age because it was necessary for these animals to grow to adulthood. We therefore chose to estimate nephron number in female 
littermates. As expected, we found that the female LPD offspring at 4 weeks of age had significant reductions in nephron number when compared with NPD offspring. These results are consistent with previous estimates of nephron number from our laboratory in male LPD offspring at 4 weeks of age [5]. This is also in accordance with other previous reports of no difference in nephron endowment between males and females in rat [31] and human studies $[2,32]$. In another recent study in rats, in which nephron number was investigated in both male and female offspring, following maternal protein restriction during pregnancy they found the reduction in nephron endowment tended to be greater in males [33].

In the Brenner hypothesis, one of the key links between reduced nephron endowment and renal disease is systemic hypertension [18]. In a carefully conducted stereological autopsy analysis of kidneys, Keller et al. demonstrated that patients with primary hypertension had fewer glomeruli per kidney than their normotensive controls [34]. In addition, developmental nephron deficit and/or maternal protein restriction in rats has been linked to the development of adult onset hypertension [3, 31, 35]. However, consistent with our previous reports [5], in the present study there was no effect of maternal protein restriction (and thus reduced nephron endowment) on conscious mean arterial blood pressure in adulthood. This finding probably reflects our deliberate choice of normotensive WKY rats in the present study, which show resistance to hypertension. Indeed, cross-suckling pups of spontaneously hypertensive rats with WKY dams do not develop hypertension [36]. It was hoped that by excluding hypertension, which is a known independent contributor to renal fibrosis and is associated with renal AGE accumulation [37], we would be able to examine the independent effect of AGE accumulation on secondary renal injury. Certainly, hypertension and AGE accumulation appear to have synergistic effects, as demonstrated by accelerated nephropathy in spontaneously hypertensive rats with diabetes, in which a combination of blood pressure reduction and inhibition of AGE formation have greater benefit than either therapy alone [37].

In accordance with the lack of effect on blood pressure in WKY rats, the reduction in nephron endowment in LPD offspring in the present study did not lead to overt renal dysfunction in adulthood. Although total GFR was reduced in the LPD offspring, this is to be expected as these rats were significantly smaller than the controls. Hence, when adjusted for kidney weight, GFR was not significantly different in the LPD and NPD offspring and so there was no evidence to suggest glomerular hyperfiltration was occurring. Woods et al. $[33,35]$ have also investigated the effects of maternal protein restriction on renal function in adulthood in Sprague-Dawley rats. As in the present study, the LPD offspring had low birth weight and smaller kidneys with fewer glomeruli when compared with control NPD offspring. In addition, although there was an increase in mean glomerular volume in LPD offspring, there was no evidence to suggest that glomeruli were hyperfiltering. Indeed, the GFR per gram of kidney weight was reduced compared with NPD controls [35], possibly reflecting the $10 \mathrm{mmHg}$ increase in mean arterial pressure in the LPD offspring.

A number of studies have suggested that renal AGE accumulation is positively linked to renal dysfunction associated with diabetes and ageing [29, 30]. Our findings demonstrate that the kidneys of LPD offspring, in which there was a $28 \%$ reduction in nephron number, are more susceptible to AGE accumulation during normal ageing and accumulate AGEs more rapidly following exposure to increased circulating levels of AGEs, possibly via changes in AGE clearance receptors [38]. Moreover, an increased fibrogenic response to AGE-BSA was observed in the kidneys of LPD offspring. It is possible to speculate that in the presence of high circulating AGEs such as is seen in diabetes mellitus, kidneys with developmental or acquired nephron deficits may be more vulnerable to AGE-mediated damage. Certainly, the rate of progression of diabetic renal disease is not linear, but rather accelerates with additional renal injury.

Moreover, our study is consistent with findings showing that the rate of progression of diabetic renal disease is faster in patients with a single kidney [39]. Although this has been argued to represent the role of glomerular hyperfiltration in the development and progression of diabetic renal injury, it is possible that AGEs may also be involved in linking nephron depletion and progressive renal damage.

In summary, our findings demonstrate that the adult kidney associated with a congenital nephron deficit is more vulnerable to a secondary renal insult following an infusion of AGE-BSA. Since AGE formation is markedly elevated with hyperglycaemia, our findings suggest that kidneys with a reduced nephron endowment may be more susceptible to diabetic renal disease.

Acknowledgements The authors gratefully acknowledge the technical assistance of R. Widdop, E. Jones, R. Flower and C. Tikellis. This study was presented in abstract form at the annual meeting in 2003 of the Australian and New Zealand Society of Nephrology and The Seventeenth National Workshop on Fetal and Neonatal Physiology. The studies were supported by funding from the National Health and Medical Research Council of Australia and Monash University, Australia.

\section{References}

1. Hinchliffe SA, Lynch MR, Sargent PH, Howard CV, Van Velzen D (1992) The effect of intrauterine growth retardation on the development of renal nephrons. Br J Obstet Gynaecol 99:296-301

2. Hughson M, Farris AB 3rd, Douglas-Denton R, Hoy WE, Bertram JF (2003) Glomerular number and size in autopsy kidneys: the relationship to birth weight. Kidney Int 63:2113-2122

3. Langley-Evans SC, Welham SJ, Jackson AA (1999) Fetal exposure to a maternal low protein diet impairs nephrogenesis and promotes hypertension in the rat. Life Sci 64:965-974

4. Merlet-Benichou C, Gilbert T, Muffat-Joly M, LelievrePegorier M, Leroy B (1994) Intrauterine growth retardation leads to a permanent nephron deficit in the rat. Pediatr Nephrol $8: 175-180$ 
5. Zimanyi MA, Bertram JF, Black MJ (2004) Does a nephron deficit in rats predispose to salt-sensitive hypertension? Kidney Blood Press Res 27:239-247

6. Nenov VD, Taal MW, Sakharova OV, Brenner BM (2000) Multi-hit nature of chronic renal disease. Curr Opin Nephrol Hypertens 9:85-97

7. Vlassara H, Striker LJ, Teichberg S et al. (1994) Advanced glycation end products induce glomerular sclerosis and albuminuria in normal rats. Proc Natl Acad Sci USA 91:11704-11708

8. Verbeke P, Perichon M, Borot-Laloi C, Schaeverbeke J, Bakala H (1997) Accumulation of advanced glycation endproducts in the rat nephron: link with circulating AGEs during aging. J Histochem Cytochem 45:1059-1068

9. Verzijl N, DeGroot J, Thorpe SR et al. (2000) Effect of collagen turnover on the accumulation of advanced glycation end products. J Biol Chem 275:39027-39031

10. Sebekova K, Podracka L, Blazicek P et al. (2001) Plasma levels of advanced glycation end products in children with renal disease. Pediatr Nephrol 16:1105-1112

11. Vlassara H, Palace MR (2002) Diabetes and advanced glycation end products. J Intern Med 251:87-101

12. Makita Z, Radoff S, Rayfield EJ et al. (1991) Advanced glycosylation end products in patients with diabetic nephropathy. N Engl J Med 325:836-842

13. Forbes JM, Thomas MC, Thorpe SR, Alderson NL, Cooper ME (2004) The effects of valsartan on the accumulation of circulating and renal advanced glycation end products in experimental diabetes. Kidney Int Suppl: S105-S107

14. Forbes JM, Thorpe SR, Thallas-Bonke V et al. (2005) Modulation of soluble receptor for advanced glycation end products by angiotensin-converting enzyme-1 inhibition in diabetic nephropathy. J Am Soc Nephrol 16:2363-2372

15. Forbes JM, Yee LT, Thallas V et al. (2004) Advanced glycation end product interventions reduce diabetes-accelerated atherosclerosis. Diabetes 53:1813-1823

16. Uribarri J, Peppa M, Cai W et al. (2003) Restriction of dietary glycotoxins reduces excessive advanced glycation end products in renal failure patients. J Am Soc Nephrol 14:728-731

17. Doi T, Vlassara H, Kirstein M et al. (1992) Receptor-specific increase in extracellular matrix production in mouse mesangial cells by advanced glycosylation end products is mediated via platelet-derived growth factor. Proc Natl Acad Sci USA 89:2873-2877

18. Brenner BM, Lawler EV, Mackenzie HS (1996) The hyperfiltration theory: a paradigm shift in nephrology. Kidney Int 49:1774-1777

19. Oldfield MD, Bach LA, Forbes JM et al. (2001) Advanced glycation end products cause epithelial-myofibroblast transdifferentiation via the receptor for advanced glycation end products (RAGE). J Clin Invest 108:1853-1863

20. Thomas MC, Cooper ME, Forbes JM (2005) The accumulation of low molecular weight fluorphores in diabetes. Ann NY Acad Sci 1043:1-11

21. Black MJ, Briscoe TA, Constantinou M, Kett MM, Bertram JF (2004) Is there an association between level of adult blood pressure and nephron number or renal filtration surface area? Kidney Int 65:582-588
22. Shweta A, Denton KM, Kett MM et al. (2005) Paradoxical structural effects in the unilaterally denervated spontaneously hypertensive rat kidney. J Hypertens 23:851-859

23. Zilin S, Naifeng L, Bicheng L, Jiping W (2001) The determination of AGE-peptides by flow injection assay, a practical marker of diabetic nephropathy. Clin Chim Acta 313:69-75

24. Stegemann H, Stalder K (1967) Determination of hydroxyproline. Clin Chim Acta 18:267-273

25. Forbes JM, Thallas V, Thomas MC et al. (2003) The breakdown of preexisting advanced glycation end products is associated with reduced renal fibrosis in experimental diabetes. Faseb J 17:1762-1764

26. Tikellis C, Johnston CI, Forbes JM et al. (2003) Characterization of renal angiotensin-converting enzyme 2 in diabetic nephropathy. Hypertension 41:392-397

27. Forbes JM, Soulis T, Thallas V et al. (2001) Renoprotective effects of a novel inhibitor of advanced glycation. Diabetologia 44:108-114

28. Jones SE, Bilous RW, Flyvbjerg A, Marshall SM (2001) Intrauterine environment influences glomerular number and the acute renal adaptation to experimental diabetes. Diabetologia 44:721-728

29. Forbes JM, Cooper ME, Thallas V et al. (2002) Reduction of the accumulation of advanced glycation end products by ACE inhibition in experimental diabetic nephropathy. Diabetes 51:3274-3282

30. Karachalias N, Babaei-Jadidi R, Ahmed N, Thornalley PJ (2003) Accumulation of fructosyl-lysine and advanced glycation end products in the kidney, retina and peripheral nerve of streptozotocin-induced diabetic rats. Biochem Soc Trans 31:1423-1425

31. Vehaskari VM, Aviles DH, Manning J (2001) Prenatal programming of adult hypertension in the rat. Kidney Int $59: 238-245$

32. Neugarten J, Kasiske B, Silbiger SR, Nyengaard JR (2002) Effects of sex on renal structure. Nephron 90:139-144

33. Woods LL, Weeks DA, Rasch R (2004) Programming of adult blood pressure by maternal protein restriction: role of nephrogenesis. Kidney Int 65:1339-1348

34. Keller G, Zimmer G, Mall G, Ritz E, Amann K (2003) Nephron number in patients with primary hypertension. N Engl $\mathrm{J}$ Med 348:101-108

35. Woods LL, Ingelfinger JR, Nyengaard JR, Rasch R (2001) Maternal protein restriction suppresses the newborn reninangiotensin system and programs adult hypertension in rats. Pediatr Res 49:460-467

36. McCarty R, Lee JH (1996) Maternal influences on adult blood pressure of SHRs: a single pup cross-fostering study. Physiol Behav 59:71-75

37. Davis BJ, Forbes JM, Thomas MC et al. (2004) Superior renoprotective effects of combination therapy with ACE and AGE inhibition in the diabetic spontaneously hypertensive rat. Diabetologia 47:89-97

38. Vlassara H (2001) The AGE-receptor in the pathogenesis of diabetic complications. Diabetes Metab Res Rev 17:436-443

39. Silveiro SP, da Costa LA, Beck MO, Gross JL (1998) Urinary albumin excretion rate and glomerular filtration rate in singlekidney type 2 diabetic patients. Diabetes Care 21:1521-1524 